\title{
Variations in the starting date of the pre-summer rainy season in South China, 1736-2010
}

\author{
DING Lingling ${ }^{1,2}$, GE Quansheng², ZHENG Jingyun" ${ }^{2}{ }^{*}$ HAO Zhixin ${ }^{2,3}$ \\ 1. Hubei University of Arts and Science, Xiangyang 441053, Hubei, China; \\ 2. Institute of Geographic Sciences and Natural Resources Research, CAS, Beijing 100101, China; \\ 3. Jiangsu Collaborative Innovation Center for Climate Change, Nanjing 210023, China
}

\begin{abstract}
The starting dates of the pre-summer rainy season during historical times (17361911) in Fuzhou and Guangzhou of South China, were determined and reconstructed on the basis of historical documents in the Yu-Xue-Fen-Cun archive, together with observed features of precipitation during the pre-summer rainy season. In addition, starting dates of the pre-summer rainy season from 1953 in Fuzhou and from 1952 in Guangzhou were reconstructed for the instrumental period. These data allowed for analyses of inter-annual and inter-decadal changes in the starting dates of the pre-summer rainy season in South China over the past 300 years. Results show that the mean starting date of the pre-summer rainy season in South China was the first pentad of May; in addition, periodicities in the starting dates of 2-3 years, 10 years, and 40 years were detected during the period 1736-1911, and of 2-3 years, 10 years, and 22 years during the instrumental period. From 1736 to 1911 , the earliest starting dates at Fuzhou and Guangzhou both occurred at the fourth pentad of April, while the latest starting dates were at the sixth pentad of May in Fuzhou and the first pentad of June in Guangzhou. During the instrumental period, the earliest and latest starting dates were at the fourth pentad of April and the first pentad of June, respectively, in both Fuzhou during 1953-2010 and Guangzhou during 1952-2010. The maximum difference between neighboring decades during 1736-1911 was 2.2 and 1.6 pentads in Fuzhou and Guangzhou, respectively, and during the instrumental period it was 2.5 and 2.4 pentads in Fuzhou and Guangzhou, respectively.
\end{abstract}

Keywords: South China; starting date of the pre-summer rainy season; reconstruction; variation; Yu-XueFen-Cun

\section{Introduction}

South China is the region with most precipitation in China, having the maximum rainfall in May-June and July-August (Lu, 1999). The first rainy period (May-June), also called as the

Received: 2014-01-24 Accepted: 2014-02-12

Foundation: Strategic Priority Research Program of the Chinese Academy of Sciences, No.XDA05080100; "135" strategic Research Project of IGSNRR, CAS, No.2012ZD001; Basic Research Project of the Ministry of Science and Technology, No.2011FY120300.

Author: Ding Lingling (1984-), PhD, specialized in climate changes. E-mail: linglingding@gmail.com

"Corresponding author: Hao Zhixin (1975-), PhD, specialized in climate changes. E-mail: haozx@igsnrr.ac.cn 
pre-summer rainy season, comprises more than $30 \%$ of the total annual precipitation. The pre-summer rainy season in South China is characterized by heavier, longer-lasting, and more frequent rainfall, as well as more flooding events. The pre-summer rainy season is the first stage that the monsoon rainband occurs and starts northward from South China (Tao and Chen, 1987; Ding and Zhang, 2009). Therefore, studies on the characteristics of the starting time of the pre-summer rainy season in South China will not only favour our further understanding of the features of precipitation in South China, but also is important to the study on the relationship between variations in the pre-summer rainy season in South China and those of the monsoon in eastern China, as well as the more general features of precipitation in China.

The onset of the pre-summer rainy season in South China has varied annually over the past 50 years (Qiang and Yang, 2008; Zhang et al., 2008; Ding et al., 2010; Li et al., 2010; Qiang and Yang, 2010). For example, based on precipitation records since 1957, Ding et al. (2010) and Qiang and Yang (2008) showed that the earliest and latest starting dates of the pre-summer rainy season in South China was the first pentad of March and the first pentad of June, respectively; in addition, they found that inter-annual variability in the starting date of the pre-summer rainy season occurred in quasi-periodic cycles of 2-8 years on inter-annual scales and 10 years on inter-decadal scales. However, divergent views on inter-annual and inter-decadal variability exist, as the duration of most data series is limited to the instrumental data period of the past 50 years. For example, a study by Ding et al. (2010) showed that the onset date of the pre-summer rainy season in South China tended to be earlier during 1957-2001, while the study of Qiang and Yang (2008) showed that the onset date tended to be later during 1957-2004. In addition, different studies have come to different conclusions regarding trends at inter-decadal scales. Therefore, longer time series of data on the starting date of the pre-summer rainy season in South China are required for the study of inter-decadal variability and long-term variations.

Based on the extracted climate information from the historical records of the $Y u$ (rainfall)-Xue (snowfall)-Fen (length unit, $0.32 \mathrm{~cm}$ approximately)-Cun (3.2 cm approximately) in the Qing Dynasty, climate records (such as the starting time of the rainy season) have been reconstructed for Beijing and Shanghai (Wang et al., 2008), Kunming (Yang et al., 2006), the middle and lower reaches of the Yangtze River (Ge et al., 2007), and the middle reaches of the Yellow River (Ge et al., 2010; Pan et al., 2013). In addition, we have designed methods for reconstructing the starting date of the pre-summer rainy season using the characteristics of the content and form of the Yu-Xue-Fen-Cun records in Fuzhou in the Qing Dynasty, using precipitation features during March-July obtained from instrumental data in Fuzhou during the period 1961-2010 (Ge et al., 2011). While only part of the $\mathrm{Yu}$-Xue-Fen-Cun records in South China are as complete as those in Beijing and Shanghai (Wang et al., 2008), which contain daily rainfall infiltration in units of Fen and Cun, other detailed precipitation information is available in the $\mathrm{Yu}$-Xue-Fen-Cun records of Fuzhou and Guangzhou. Thus, our goal is to reconstruct a series of starting dates for the pre-summer rainy season in Fuzhou and Guangzhou since 1736, using data from the Yu-Xue-Fen-Cun archive, and to use the series to analyze patterns of inter-annual and inter-decadal variability in this region. 


\section{Data and reconstruction methods}

\subsection{Data}

The Yu-Xue-Fen-Cun records for the period 1736-1911 during the Qing Dynasty (1644-1911) provide the main material for our study. The data were extracted from the original archives, such as memos with Emperor's red postil and duplicate memos from the Military Department (which were reported by local government officials, administration officials, military officials, salt administration officials, river management officials, education commissioner officials, and so on), which were kept in the First Historical Archives of China. We extracted a total of 1417 records from Fuzhou and 1212 records from Guangzhou. Records of daily precipitation amounts are difficult to be recorded in the absence of modern observation, especially as precipitation in South China is characterized by heavy and long-lasting rainfall events. Therefore, except for a few records in the Yu-Xue-Fen-Cun of South China, which include daily precipitation infiltration in units of Fen and Cun, most records only give evaluative descriptions of the amount of precipitation within certain periods, including the date and intensity of rainfall events, the number of rainy days, and descriptions of rainfall and related conditions, such as natural disasters and losses, agricultural harvests, and so on.

The records from the Yu-Xue-Fen-Cun archive in Fuzhou and Guangzhou can be divided into five classes according to contents and recording types. The first class (I) includes records of daily precipitation infiltration in units of Fen and Cun, including rainfall dates and infiltration depths based on quantitative information. For instance, Song Shou, the Governor General of Fujian and Zhejiang, reported "Minxian county in Fuzhou Fu received 1 Fen of rainfall on the 2 nd day of the third month (30/4 $)$ of the Chinese lunar calendar, and 1 Fen on the 7 th day $(5 / 5)^{2,}$ " during the 3rd year of Xuantong Emperor (1911). The second class (II) includes records of dates of rainfall and evaluative descriptions of the precipitation intensity. For instance, Jueluo Jiqing, the Governor General of Guangdong, reported "Provincial Capital received moderate rain on the 8 th and 11 th days of the $3 r d$ month $(8 / 4,11 / 4)$ in the Chinese lunar calendar" during the 5th year of Jiaqing Emperor (1800). The third class (III) includes records of precipitation dates within certain periods (usually in the form of a month in the lunar calendar), including the numbers of rainy days within certain periods. For instance, $\mathrm{Xu}$ Jishe, the Governor General of Fujian, reported "Provincial Capital received rain on the 7th, 10th, 18th, 19th, 22nd, and 28th days of the 2 nd month $(1 / 3,4 / 3,12 / 3,13 / 3,16 / 3$, 22/3) in the Chinese lunar calendar" during the 29th year of Daoguang Emperor (1849). The fourth class (IV) includes records of the precipitation duration within certain periods (usually in the form of a month or a period of 10 days in the lunar calendar). For instance, Qing Rui, the Governor General of Fujian, reported "Provincial Capital was fine during the

\footnotetext{
1 30/4 denotes April 30 in the Gregorian calendar.

${ }^{2}$ Songshou, Governor General of Fujian and Zhejiang. Duplicate memos by the Military Department, on the 22nd day of the 5th month of the lunar calendar, during the 3rd year of Emperor Xuantong (18 June 1911).

3 Jueluo Jiqing, Governor General of Guangdong. Memos by the Emperor with red postil, on the 3rd day of the 4th month of the lunar calendar, during the 5th year of Emperor Jiaqing (26 April 1800)

${ }^{4} \mathrm{Xu}$ Jishe, Governor General of Fujian. Memos by the Emperor with red postil, on the 28th day of the 3rd month of the lunar calendar, during the 29th year of Emperor Daoguang (20 April 1849).
} 
1 st ten days of the $3 \mathrm{rd}$ month (3/4-12/4), and totally received rain 8 times during the 2 nd and $3 r d$ ten days of the 3rd month (13/4-2/5) of lunar calendar" during the 9th year of Xianfeng Emperor (1859). The fifth class (V) includes records of evaluative descriptions of the amount of precipitation within certain periods, from which information can be obtained about the intensities of rainfall and rainfall anomalies occurring during certain periods. For instance, Lao Chongguang, the Governor General of Guangdong, reported "Provincial Capital in Guangdong received less rain and more fine days in the 4th month (29/4-27/5) of the lunar calendar ${ }^{6}$ " during the 1st year of Tongzhi Emperor (1862). The number and the proportion of each class are represented in Table 1 . The records that include the dates and amounts or intensities of rainfall (first and second class records) comprise $22.4 \%$ of the total number of Yu-Xue-Fen-Cun records in Fuzhou and Guangzhou; the records which include dates and times of rainfall within certain periods (third and fourth class records) comprise $16.2 \%$ of the total number of records. These quantitative records provide important evidence for identifying the starting time of the pre-summer rainy season, and the same as the fifth class records (61.4\% of the total number of records), since these qualitative records contain valuable information about occurrences of rainfall anomalies and the intensities of anomalies within certain periods (bounded by definite starting and ending dates).

Table 1 Total pieces for each recording format categories from the Yu-Xue-Fen-Cun records in Fuzhou and Guangzhou, 1736-1911

\begin{tabular}{ccccccc}
\hline Sites & I & II & III & IV & V & Total \\
\hline Fuzhou & 6 & 305 & 119 & 272 & 715 & 1417 \\
Guangzhou & 0 & 278 & 20 & 14 & 900 & 1212 \\
Total & 6 & 583 & 139 & 286 & 1615 & 2629 \\
Proportion (\%) & 0.2 & 22.2 & 5.3 & 10.9 & 61.4 & 100.0 \\
\hline
\end{tabular}

In addition, we collected drought and flood disaster records (38 records in Fuzhou and 301 records in Guangzhou) from the Collection of the Climate Records in China during the Three Thousand Years (Zhang, 2004) and the China Meteorological Disaster Canon (Ding, 2008) to supplement years which were lacking in the Yu-Xue-Fen-Cun records. Also, a few drought/flood disasters ( 5 pieces in Fuzhou and 68 pieces in Guangzhou) during the period of Republic of China (1912-1948) were obtained from the China Meteorological Disaster Canon. However, the starting times of the rainy seasons could be identified in only a few of the years ( 1 year in Fuzhou and 6 years in Guangzhou) and it was difficult to supplement the data in other years. Therefore, the reconstructed series during this period was incomplete.

The instrumental data are downloaded from the website of http://cdc.cma.gov. cn/home.do, which has been quality controlled by Chinese Meteorological Administration. The dataset includes 67 sites in South China with complete daily precipitation records from April to September during the period 1961-2010; while the records in Fuzhou and Guangzhou began in 1953 and 1952, respectively. To analyze the features of the pre-summer rainy

\footnotetext{
${ }^{5}$ Qing Rui, Governor General of Fujian. Duplicate memos by the Military Department, on the 27th day of the 4th month of the lunar calendar, during the 9th year of Emperor Xianfeng (29 May 1859).

${ }^{6}$ Lao Chongguang, Governor General of Guangdong. New directory, on the 30th day of the 5th month of the lunar calendar, during the 1st year of Emperor Tongzhi (26 June 1862).
} 
season in South China, and the spatial representiveness of Fuzhou and Guangzhou to the whole South China region, we calculated the correlation coefficients of series of April-September daily precipitation among the 67 stations from 1961 to 2010, when the correlation coefficients are $\geq 0.2$ (statistical significance, $p<0.01), 0.4$, and $0.5(p<0.001)$, they occupy $50 \%, 25 \%$ and $12.5 \%$ of the total number of selected stations, respectively. Based on the stepwise remove method, 40 representative stations (16 in Guangdong, 13 in Fujian, and 11 in Guangxi) can be used to analyze the features of precipitation in South China. As precipitation amounts are influenced by both mesoscale and large-scale circulation systems, as well as local geographical features, the variations among stations in South China are not completely consistent. However, the number of stations where the correlation coefficient between other stations and Fuzhou is greater than 0.2, 0.4 and 0.5, is 32, 14 and 8, respectively, and that is 39,15 , and 7 for Guangzhou, respectively, indicating that the rainfall records in Fuzhou and Guangzhou can better represent the whole of South China to a certain extent.

\subsection{Reconstruction methods}

The annual precipitation pattern in South China is characterized by a double-peaked curve (Lu, 1999; Ge et al., 2011), according to analyses of modern daily instrumental data. Variations in precipitation in South China during the first half year show that precipitation begins to increase at the beginning of March, indicating the South China entered into the spring rainy season. Precipitation amount is stable from the second 10 days in March to the second 10 days in April, and after that, it begins to decrease during the third 10 days in April, and then greatly increases at the beginning of May, with maximum rainfall occurring in the mid-late June, which formed the May-June pre-summer rainy season. Precipitation decreases from the third 10 days in June (at the end of the pre-summer rainy season) and reaches a minimum point at the beginning of July, when the summer drought starts.

The pre-summer rainy season precipitation is known as frontal rainfall, as it forms at a convergence zone of cold air from the north and moist warm air from a low-latitude area located from the region of Nanling to the Wuyi Mountains. The pre-summer rainy season is controlled by a blocking high of the westerlies, a subtropical high over the North Pacific, and a Bay of Bengal trough indicating the Indian southwest monsoon is a result of the onset of the southwest and southeast monsoons; it is characterized by a strong front, strong rainfall intensity, frequent rainstorms, and abundant rainfall (the precipitation amount is over $30 \%$ of the year's total; Lu, 1999). In particular, the number of moderate rainy days and heavy rainy days during the pre-summer rainy season is greater than that during the period of spring rainy season. For instance, the number of rainy days with daily precipitation $\geqslant 25 \mathrm{~mm}$ during the pre-summer rainy season is more than double that occurs during the period of persistent spring rains (Ge et al., 2011).

The features showed above provide quantitative evidences for identifying the starting date of the pre-summer rainy season. Together with the features of the Yu-Xue-Fen-Cun records in Fuzhou during the Qing Dynasty, we have defined two parameters for identifying the pre-summer rainy season in Fuzhou: a sharp increase in rainfall intensity, and a "dry season" between the spring rainy season and the pre-summer rainy season. The criteria for identifying a sharp increase in rainfall intensity are either (1) cumulative precipitation over two con- 
tinuous 5-day periods (spacing by a dry day at most) is not less than the pentad mean precipitation (PMP) during April-September, 1961-2010; or (2) cumulative precipitation during a continuous 10-day period is more than three times of the PMP. While, the dry season is identified by the number of days during which daily precipitation is not less than $10 \mathrm{~mm}$ or at most two days have greater than $5 \mathrm{~mm}$ daily precipitation (which is nearly equal to the PMP in Fuzhou of $5.4 \mathrm{~mm}$ ) during any continuous 10-day period. The method for establishing the starting date of the pre-summer rainy season in Fuzhou is as follows: from 16 April to the day on which the cumulative precipitation of any continuous 5 days is not less than $26.9 \mathrm{~mm}$ (which is the mean pentad value in climatology), and after 5-6 days of this date, the cumulative precipitation during a continuous 5-day interval is not less than $26.9 \mathrm{~mm}$, or since this date, the cumulative precipitation during a continuous 10-day interval is not less than $80.7 \mathrm{~mm}$ (three times of the PMP in Fuzhou); and, to 10 May, the number of days is more than 1 day when daily precipitation is not less than $10 \mathrm{~mm}$ during any continuous 10-day period or more than two days when daily precipitation is not less than $5 \mathrm{~mm}$. Then, this date or the first day with greater than $5.4 \mathrm{~mm}$ daily precipitation can be identified as the starting date of the pre-summer rainy season. However, if this day does not occur until the end of June, then we consider this year as empty pre-summer rainy season.

These criteria require modification when used to identify the pre-summer rainy season in Guangzhou, as precipitation during the pre-summer rainy season in Guangzhou is not as concentrated as that in Fuzhou, and the daily precipitation in Guangzhou is not equal to that in Fuzhou. First, the starting date for identification purposes is changed to 21 April instead of 16 April, and the ending date is set to 20 June, as the starting date of the pre-summer rainy season in Fuzhou and Guangzhou is no later than 20 June according to instrumental data. Second, the criteria for the brief period of rain is not less than the PMP, and is instead set as the daily precipitation in two days at most is lower than the mean value of April to September. The improved methods are as follows: From 21 April to the day on which the cumulative precipitation during a continuous 5-day period is not less than PMP, and the cumulative precipitation during two continuous 5-day periods (with a dry interval of not more than 5 days) is greater than the PMP in a consecutive 15-day period, or, the cumulative precipitation during 10 continuous days is not less than 3 times of PMP; and, to 10 May, the number of days when precipitation is not less than the PMP during any continuous 10-day period is not more than 2 . Then, this day or adjacent day when the first daily precipitation is greater than the mean value from April to September can be identified as the starting date of the pre-summer rainy season. The year can be considered as empty pre-summer rainy season if the day cannot be found until 20 June. Based on these criteria, series of starting dates of the pre-summer rainy season in Fuzhou and Guangzhou during instrumental periods can be reconstructed using daily precipitation records.

Then, in terms of the parameters of the precipitation characteristics recorded in the $Y u$-Xue-Fen-Cun archive, we established the following criteria for quantitatively identifying the sharp increase in rainfall intensity and the dry period.

(1) For years in which daily rainfall amounts are available in units of Fen or Cun, the dry season is identified firstly according to the daily rainfall amount; then, the starting date of the pre-summer rainy season is identified on the basis of a sharp increase in rainfall intensity. That is, the day when the cumulative rainfall amount during two continuous 5-day periods 
(with a dry interval of not more than 5 days) is not less than the PMP from March-July, or, the cumulative rainfall during 10 continuous days is not less than 3 times of PMP. Then, the starting date of the pre-summer rainy season is the day of the first 5 continuous days or adjacent day when the daily rainfall amount is not less than the mean value from March to July.

(2) For years with data on rainfall and intensity records, the dry season is identified from the contents of the Yu-Xue-Fen-Cun. First, the starting date of the pre-summer rainy season is identified using surrogate indices of precipitation amounts, which are based on descriptions of rainfall intensity. The surrogate indices for PMP are 1 day of rainstorm or 2 days of rain ( 1 day of heavy rain or 2 days of moderate rain), 3 days of non-light rain (at least 1 day of moderate rain), or 4 days of non-light rain. We identified rainstorm, heavy rain, moderate rain, and non-light rain according to the described phrases in the Yu-Xue-Fen-Cun. For example, they are corresponding to the "downfall lasting one day and heavy rainstorm", "persisting rain during day and night", "soaking rain, showers and continuous dense rain", “dense rain, opportune rainfall, light rain lasting one day", respectively.

(3) For years having rainfall dates or rainfall times within certain periods, the number of days or times with rainfall are counted for each month of the Chinese lunar calendar. First, rainy months and less-rain months are identified by comparing the number of rainfall days or times between neighboring months; then, the starting pentad of the pre-summer rainy season is identified according to the starting time of continuous rain in a rainy month. Although the difference between the number of rainfall days or times during persistent spring rain and that of the pre-summer rainy season is very small, the number of rainfall days or times during the less rain month is actually less than that in the starting month of the pre-summer rainy season. In general, the month must have not less than 14 rainfall days or times, since the number of days of precipitation with more than $1 \mathrm{~mm}^{7}$ is less than 14 days in any continuous 30-day period from March to July during the instrumental period from 1961 to 2010 .

Then, the first rainy month in the pre-summer rainy season, which is that with a larger number of rainfall days or times compared with neighboring months, should meet another condition; that is, the number of rainfall days or times is not less than 14. For these years, if the number of rainfall days or times of the lunar month that includes 21 April is larger than that after two months and not less than 14 days, then the pentad containing the first day with continuous precipitation after 21 April is the starting pentad of the pre-summer rainy season. Alternatively, if there is no record with rainfall dates, the starting pentad of the pre-summer rainy season is identified as the fifth pentad of April. Also, if the number of rainfall days or times of the month in the lunar calendar that includes 21 April is less than $80 \%$ of that in the next month in the lunar calendar, then this month is identified as the less-rain month, and the pentad that includes the first day of continuous precipitation in the next month is the starting pentad of the pre-summer rainy season. Or, when there is no record with rainfall dates, the starting pentad of the pre-summer rainy season is identified as the pentad that includes the

\footnotetext{
7 We chose $1 \mathrm{~mm}$ as a standard for identifying a rainy day, as soft rain with rainfall amounts less than $1 \mathrm{~mm}$ was not generally recorded in South China during the Qing Dynasty, and the average number of rainy days in a month of the lunar calendar computed using a 1-mm standard is nearly equal to values measured during the instrumental period (1961-2010), as well as the mean number of rainfall days during years with complete records in historical documents.
} 
5 th day of the next month in the lunar calendar.

(4) For years with complete descriptive records of rainfall conditions, the dry season or the rainy period during April-June can be identified firstly, and then the starting pentad of the pre-summer rainy season was recognized during the rainy period. However, when the dry or rainy season cannot be judged by the Yu-Xue-Fen-Cun archive, we then make a judgement by the drought/flood disasters records. That is, from 21st April until the rainy period during which rainfall persists for more than 10 days or flood occurs, and until 20th June, if the less rainfall lasts for less than 8 days or no drought disaster occurs, then the pentad that includes the starting date of the rainy period is the starting pentad of the pre-summer rainy season. Alternatively, when the rainy season occurs during a certain month in the lunar calendar, the pentad that includes the 5th day of the month in the lunar calendar is identified as the starting pentad of the pre-summer rainy season.

(5) When the starting date of the pre-summer rainy season could not be identified from records in the $\mathrm{Yu}$-Xue-Fen-Cun, we identified the starting pentad according to drought/flood records in Fuzhou and Guangzhou. For instance, if the rainfall occurred after the drought disaster in April, then the pentad with the starting date of rainfall in the next month is identified as the starting pentad of the pre-summer rainy season. In the case of severe drought in spring and summer, the pre-summer rainy season is considered as empty year.

If records could not be identified in terms of anyone of the above five types of years, then the year is defined as unavailable data. It should be noted that recorded dates are not clear and that reports to the same rainfall event between different reporters could be inconsistent. Facing to the conflicted data, we used a median or mean value; if two median values existed, then we choose the early one. For instance, when the date occurs during the first 10 days of April in the lunar calendar, we identified the 5th day of April in lunar calendar as the staring time; when the date occurs from spring to summer, we then took the sixth pentad of April as the starting time; when the date occurs at the turning point from spring to summer, we then used the first pentad of May as the starting time of the pre-summer rainy season.

\section{Results and analysis}

As mentioned in section 2.2, the starting dates of the pre-summer rainy season during 1736-1911 (except in years without record) were obtained from Fuzhou and Guangzhou. The numbers of identified years for Fuzhou and Guangzhou are not exactly the same: 121 (68.8\% of the total) in Fuzhou and 107 (60.8\% of the total) in Guangzhou. The number of identified years in Fuzhou during 1736-1749, 1750-1799, 1800-1849, 1850-1899, and $1900-1911$ is $12,44,33,26$, and 6 , comprising $86 \%, 88 \%, 66 \%, 52 \%$, and $50 \%$ of the total number of years during each period, respectively, while the number of identified years in Guangzhou during the same periods is $11,46,17,27$, and 6 , comprising $79 \%, 92 \%, 34 \%$, $54 \%$, and $60 \%$ of the total number of years in each period, respectively. The average starting time of the pre-summer rainy season during 1736-1749, 1750-1799, 1800-1849, 1850-1899, and 1900-1911 in Fuzhou and Guangzhou is the second pentad of May, the first pentad of May, the first pentad of May, the second pentad of May, and the first pentad of May, respectively. The number of years identified in the 18th century is greater than that in the 19th and 20th centuries. Together with the series of instrumental data, the starting pentads of the 
pre-summer rainy season in Fuzhou and Guangzhou during 1736-2010 are shown in Figure 1. In addition, in order to keep the integrity of the series, for the year with missing data, we interpolated it with the mean value over 50 years.

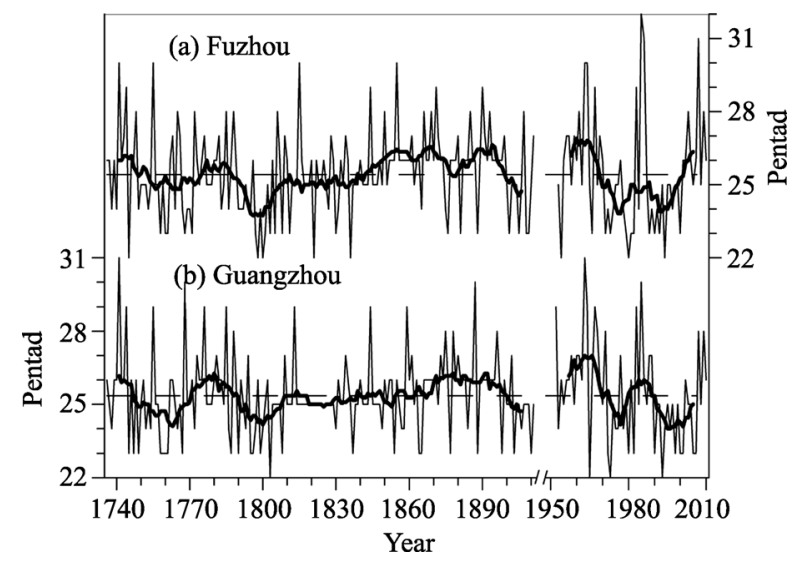

Figure 1 Starting dates of the pre-summer rainy season in Fuzhou and Guangzhou during the period 1736-2010 Note: Pentad 22, which is the 22nd pentad of the year, is equivalent to the fourth pentad of April; pentad 23 means the fifth pentad of April, and so on. The bold lines are 11-year running means; the dotted lines represent the means of the data.

\subsection{Inter-annual and inter-decadal variation}

As shown in Figure 1, during the period 1736-1911, the starting dates in the earliest and latest years in Fuzhou were the fourth pentad of April and the sixth pentad of May, respectively, while the starting dates in the earliest and latest years in Guangzhou were the fourth pentad of April and the first pentad of June, respectively. The mean starting date of the pre-summer rainy season during the period 1736-1911 was the first pentad of May in both Fuzhou and Guangzhou. And empty pre-summer rainy seasons was in 1815 in Fuzhou and in 1768 in Guangzhou. The maximum difference between the starting dates of the pre-summer rainy season in neighboring years was 7 pentads in both Fuzhou and Guangzhou. During the instrumental period, the earliest starting dates, latest starting dates, and mean starting dates were the fourth pentad of April, the first pentad of June, and the first pentad of May, respectively, in both Fuzhou and Guangzhou, with an empty pre-summer rainy season in 1985 in Fuzhou. The maximum difference between the starting dates of neighboring years in Fuzhou during 1953-2010 is 6 pentads, while the maximum difference in Guangzhou during 1952-2010 is 7 pentads. The difference between the starting date of the earliest and the latest year is 9 pentads (approximately 1.5 months). These data reveal that significant inter-annual variability exists in the starting dates of the pre-summer rainy season over South China.

On the decadal time scale, the starting date of the earliest decade from the 1740s to 1900 s was the sixth pentad of April in both Fuzhou and Guangzhou. The starting date of the latest decade was the third pentad of May in Fuzhou and the second pentad of May in Guangzhou. The mean of the starting date from the 1740s to 1900s was the first pentad of May in both Fuzhou and Guangzhou. The maximum difference between decades during the 1740s to 
1900s was 2.2 pentads (between the 1900s and 1890s) in Fuzhou and 1.6 pentads (between the 1790s and 1780s) in Guangzhou. The earliest, latest, and mean starting dates during the 1960s to 2000s were the sixth pentad of April, the third pentad of May, and the first pentad of May, respectively, in both Fuzhou and Guangzhou. The inter-decadal maximum difference from the 1960s to the 2000s was 2.5 pentads in Fuzhou and 2.4 pentads in Guangzhou (both occur between the 1970s and 1960s). The difference between the earliest and latest starting dates from the 1740 s to the 2000 s is 2.8 pentads and 3.0 pentads (approximately 0.5 months) in Fuzhou and Guangzhou, respectively. These data reveal that significant inter-decadal variability exists in the starting dates of the pre-summer rainy season over South China.

\subsection{Cycle analysis}

To analyze the variability of cycles in the starting dates of the pre-summer rainy season in South China, the spectral analysis and wavelet analysis were performed on the data during the reconstructed and instrumental period. As required for the software performing, for empty pre-summer rainy season years, the starting dates are valued as the next pentad of the starting dates in the latest year within the 50 years. For example, the starting date of the empty pre-summer rainy season of 1786 in Guangzhou was taken as the sixth pentad of May, while that of the empty rainy season of 1815 in Fuzhou was taken as the second pentad of June (Figures 2 and 3).
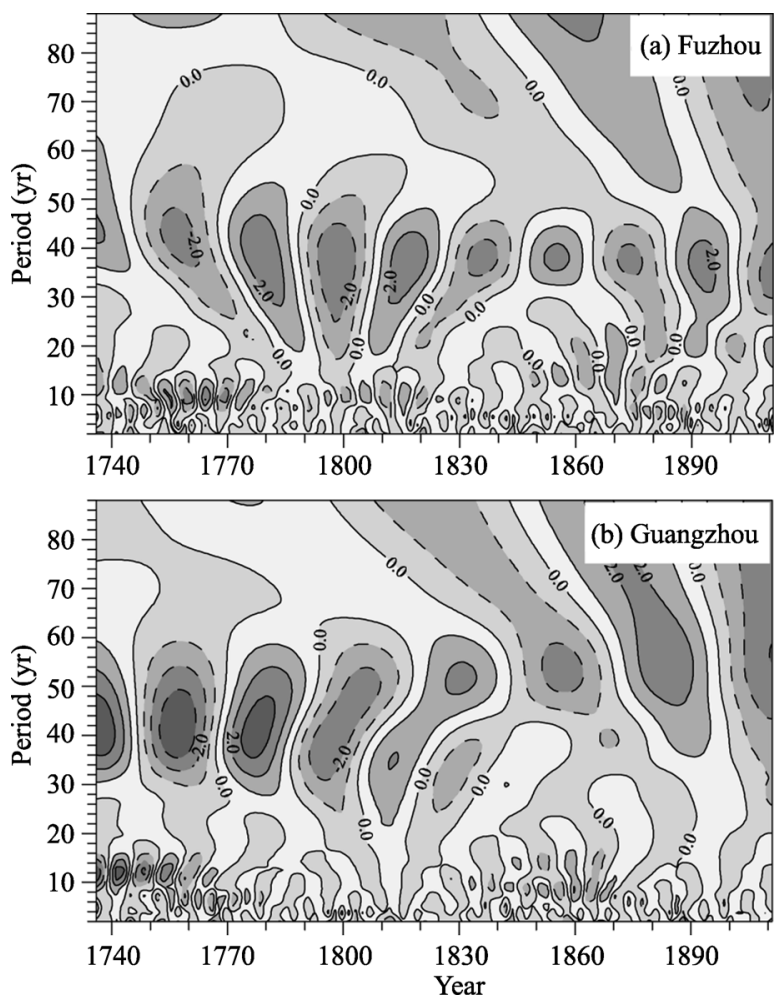

Figure 2 Wavelet transforms of the starting dates of the pre-summer rainy season in Fuzhou and Guangzhou during 1736-1911

(Note: The dotted line circled areas mean the advanced rainy season, while the solid lines circled areas mean the delayed rainy season.) 


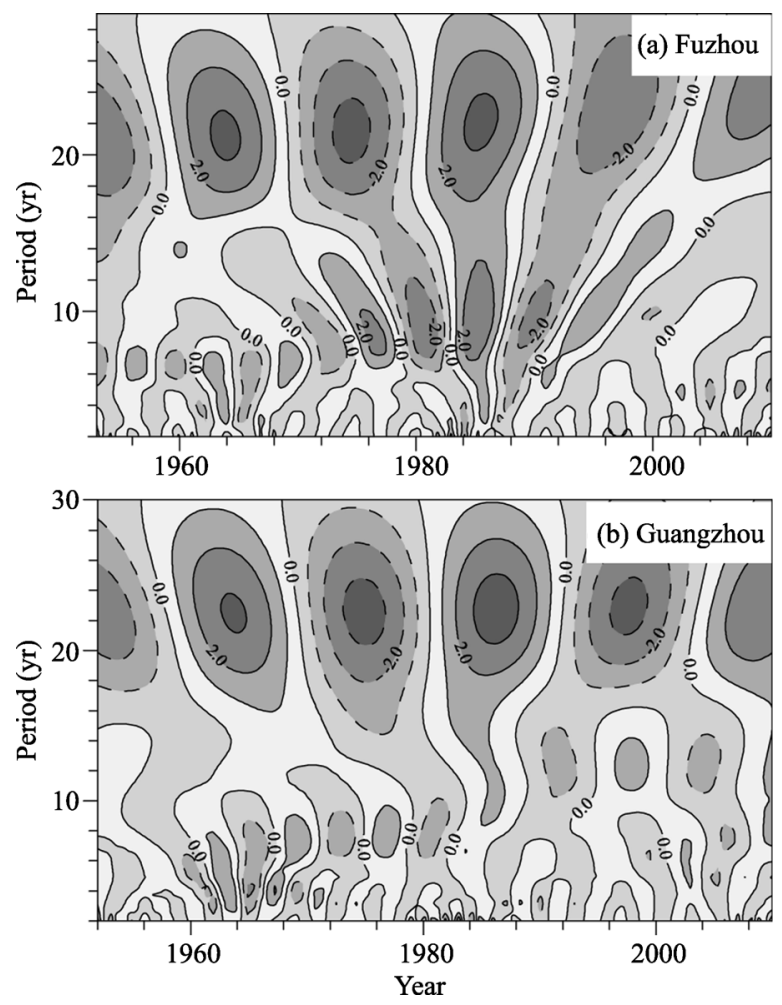

Figure 3 The same as Figure 2, but in Fuzhou during 1953-2010 and Guangzhou during 1952-2010

According to the spectral analysis of the starting date series, the most significant cycles during 1736-1911 have periodicities of 2.68 years and 2.74 years in Fuzhou; however, Guangzhou has a weaker inter-annual cycle, but a 40 -year cycle is very significant. The most significant cycles during the instrumental period are 2.71 years and 2.11 years in Fuzhou and 3.64 years in Guangzhou (did not pass 95\% confidence level).

Figures 2 and 3 illustrate the periodicities of starting dates series of the pre-summer rainy season in Fuzhou and Guangzhou during the reconstructed and instrumental periods, respectively, transformed by Morlet wavelet analysis. Both Fuzhou and Guangzhou have a quasi-cycle of 40 years during 1736-1911, and was more stable in Fuzhou. While, in Guangzhou, the 40- year cycle became weaker after 1820, and was replaced by a significant 50 -year cycle. The second significant quasi-cycle during 1736-1911 is of 10 years, which is significant at the two periods of 1736-1775 and 1790-1835 in Fuzhou, but in Guangzhou, which is replaced by a 6-8 year cycle during the period of 1830-1870. During the instrumental period, the most significant cycle is of 22 years and 6-10 years as the secondary in both Fuzhou and Guangzhou.

\section{Discussion and conclusions}

\subsection{Discussion}

The uncertainties in the results of the analyses should be discussed, as there exist a few years during 1736 to 1911 for which the starting dates of pre-summer rainy season cannot be iden- 
tified due to a lack of records in Fuzhou and Guangzhou. However, the general features of the series during the reconstructed and the instrumental periods are very consistent. For instance, (1) the mean of the starting date of the pre-summer rainy season is the first pentad of May in both periods, (2) the starting date in the earliest year and the latest year during the reconstructed period is approximately close to that during the instrumental period, and (3) there existed 2-3-year and 10-year cycles in both periods. Therefore, although uncertainties existed in the reconstructed series, these years with missing data have very little influence on the characteristics of inter-annual and inter-decadal variations in the starting time of the pre-summer rainy season in South China.

\subsection{Conclusions}

Using the Yu-Xue-Fen-Cun archive and drought/flood disasters recorded in Chinese historical documents, series of starting dates of the pre-summer rainy season in Fuzhou and Guangzhou were reconstructed for the period 1736-1911. In addition, series of starting dates of the pre-summer rainy season during the instrumental period were established and analyzed, based on data obtained from daily precipitation records in Fuzhou during 1953-2010 and in Guangzhou during 1952-2010. Inter-annual to inter-decadal variations and periodicities in the starting dates were analyzed in both the reconstructed and the instrumental datasets.

(1) The analysis of the data from the reconstructed period (1736-1911) shows that the mean, earliest starting date of the pre-summer rainy season were the first pentad of May and the fourth pentad of April, respectively, in both Fuzhou and Guangzhou, and the latest starting date was the sixth pentad of May in Fuzhou and the first pentad of June in Guangzhou. The earliest decadal starting date was the sixth pentad of April in both Fuzhou and Guangzhou, and the latest decadal starting date was the third pentad of May in Fuzhou and the second pentad of May in Guangzhou. The maximum differences between the neighboring decades were 2.2 pentads (between the 1900s and 1890s) in Fuzhou and 1.6 pentads (between the 1790s and 1780s) in Guangzhou. The 2-3-year, 10-year, and 40-year cycles were presented in series of the starting date in both Fuzhou and Guangzhou.

(2) During the instrumental period, the earliest, latest and mean starting date of the pre-summer rainy season were the fourth pentad of April, the first pentad of June, and the first pentad of May, respectively, in both Fuzhou and Guangzhou. The starting dates in the earliest and latest decades (1960s-2000s) were the sixth pentad of April and the third pentad of May in Fuzhou and Guangzhou, respectively. The maximum difference between two adjacent decades was 2.5 pentads (between the 1970s and 1960s) and 2.4 pentads (between the 1970s and 1960s) in Fuzhou and Guangzhou, respectively. There existed 2-3-year, 10-year, and 22-year cycles in both Fuzhou and Guangzhou.

(3) Generally, the mean starting date of the pre-summer rainy season from 1736 to 2010 in South China is the first pentad of May, but with obvious inter-annual (2-3 years) and inter-decadal (quasi-10 years, 22 years and 40 years) variability. The earliest and latest starting date was the fourth pentad of April and the first pentad of June, respectively, with 9 pentads (approximately 1.5 months) for amplitude (the difference between maximum and minimum values) of whole series. The maximum amplitude between neighboring years was 7 pentads. At the decadal time scale, the starting date in the earliest decade (1740s) and the latest dec- 
ade (2000s) were the sixth pentad of April and the third pentad of May, respectively, with 3 pentads for amplitude of the whole series. The maximum inter-decadal amplitudes were both more than 2 pentads in Fuzhou and Guangzhou.

\section{References}

Ding J L, Xu Z S, Fei J F et al., 2009. Analysis of the definition of the onset and ending dates of the annually first rainy season in South China and its interannual variation characteristics. Journal of Tropical Meteorology, 25(1): 59-65. (in Chinese)

Ding Y H, 2008. China Meteorological Disaster Canon. Beijing: China Meteorological Press. (in Chinese)

Ding Y H, Zhang J Y, 2009. Rainstorm and Flood. Beijing: China Meteorological Press. (in Chinese)

Ge Q S, Ding L L, Zheng J Y et al., 2011. Research on methods of starting date of pre-summer rainy season reconstruction in Fuzhou derived from Yu-Xue-Fen-Cun records. Advances in Earth Science, 26(11): 1200-1207. (in Chinese)

Ge Q S, Guo X F, Zheng J Y et al., 2007. Meiyu in the middle and lower reaches of the Yangtze River since 1736. Chinese Science Bulletin, 52(23): 2792-2797. (in Chinese)

Ge Q S, Hao Z X, Tian Y Y et al., 2010. The rainy season in the northwestern part of the East Asian summer monsoon in the 18th and 19th centuries. Quaternary International, doi: 10.1016/j.quaint.2010.02.025.

Li L P, Zhang K M, Wang C et al., 2010. Temporal and spatial variations of extreme precipitation in the pre-flood period of South China in recent 40 years. Climatic and Environmental Research, 15(4): 443-450. (in Chinese)

Lu S J, 1990. Climate in Southern China. Beijing: China Meteorological Press. (in Chinese)

Pan W, Zheng J Y, Xiao L B et al., 2013. The relationship of nature runoff changes in flood-season of middle Yellow River and Yongding River, 1766-2004. Acta Geographica Sinica, 68(7): 975-982. (in Chinese)

Qiang X M, Yang X Q, 2008. Onset and end of the first rainy season in South China. Chinese Journal of Geophysics, 51(5): 1333-1345. (in Chinese)

Qiang X M, Yang X Q, 2010. Anomalies of the first rainy season onset dates in South China and its relationship with circulation and sea surface temperature in pre-winter. Abstracts of the 8th Annual Academic Conference for PhD Candidates, 38. (in Chinese)

Tao S Y, Chen L X, 1987. A review of recent research on the East Asian summer monsoon in China. In: Chang C P, Krishnamurti T N. Monsoon Meteorology. New York: Oxford University Press: 60-92.

Wang W C, Ge Q S, Hao Z X et al., 2008. Rainy season at Beijing and Shanghai since 1736. Journal of the Meteorological Society of Japan, 86(5): 827-834.

Yang Y D, Man Z M, Zheng J Y, 2006. Reconstruction of series in later or earlier starting date of rainy season in Yunnan Province and evolvement of summer monsoon in Qing period. Acta Geographica Sinica, 61(7): 705-712. (in Chinese)

Zhang D E, 2004. Collection of the Climate Records in China during the Three Thousand Years. Nanjing: Phoenix Education Publishing. (in Chinese)

Zhang Y, Sun Z B, Bai Y Y et al., 2008. Drought/flood characteristics of pre-flood season in South China in recent 47 years. Journal of Nanjing Institute of Meteorology, 31(2): 176-182. (in Chinese) 